\title{
Evaluation of the Selected Parameters of the Body onto the Location of the Gravity Center Projected on Foot
}

\section{Sławomir Pasko ${ }^{1}$, Marek Sutkowski ${ }^{2 *}$ and Beata $\mathbf{Z u k}^{3}$}

${ }^{1}$ Institute of Micromechanics and Photonics, Warsaw University of Technology, Poland

${ }^{2}$ Institute of Microelectronics and Optoelectronics, Warsaw University of Technology, Poland

${ }^{3}$ Department of Biophysics and Physiology, Medical Faculty, Medical University of Warsaw, Poland

\begin{abstract}
The purpose of this study is to evaluate mutual influence of selected postural parameters onto movement of the line of the human body gravity point with use of noncontact optical tool (photogrammetry based). This study constitutes a part of the wider observational cross-sectional study of body posture of healthy people. A specially designed measurement set-up was developed to determine the 3D location of selected characteristic points of the human body. The photogrammetry tool was applied to calculate $(x, y, z)$ coordinates of the points marked on the examined body, e.g., processus mastoideus and angulus superior scapulae. With these assumptions postural parameters in the sagittal and coronal planes can be determined, respectively. The 3D coordinates were used to evaluate the correlation between position of the examined body points and movement of the line of the examined' body gravity point. It can be seen that it is impossible to determine the correlation of the absolute values of the angles of rotation as simple subject to analysis. To demonstrate the interdependence, it is necessary to take into account the sign of the angle of rotation. In the presented study, this factor can be analyzed. Obtained results suggest the usefulness of presented system in physiotherapy of body posture.
\end{abstract}

Keywords: Posture; Patient positioning; Physiology; Musculoskeletal systems; Optical imaging

\section{Introduction}

Monitoring the status of the locomotor system, the skeleton is a key factor in the diagnosis, monitoring and treatment of common human disorders or lesions associated with posture. Determination of deviations from accepted standards (considering the individual characteristics significant for their own anatomy and physiology of the examined) and control changes over time is the primary task of the physiotherapy study, which first objective is to seek the causes of the observed changes. A human movement system is a set of interrelated elements that make up a static body position in space and constantly undergo dynamic changes during daily activities. Keeping habitual position and perform any physical activities is a result of changes in the network connections of all components of the human kinematic motion system. As noted by Kapandji [1], Neuman [2], Chaitov [3], Błaszczyk [4] and other authors, abnormal function of one of its elements (e.g., joints), pain or skin lesion (e.g., the scar) may form an internal compensation so as to be able to move with the least possible effort of the energy. The general approach can be divided into disorders of the musculoskeletal system for static and dynamic. Static dysfunctions are associated with impaired local and general irregularities affecting the function of the supporting leg and the wrong body position [5]. In turn, the dynamic dysfunctions are related to changes and abnormalities in all functions of the body's motor (movement, grip, etc.) [6].

The research on the various parameters that define the attitude of the body using various methods of measurement are very commonly found in the literature. Among them we can distinguish a number of works containing the results of studies on the impact and dependencies of the measured values on the body parameters in relation to adopted posture.

Cheng et al. has been noticed the influence of the proper selection of chairs to stabilize the posture of children while writing [7]. Fortin et al. describes the differences in the angle of scoliosis in standing and sitting positions [8]. In turn, Annetts et al. studied subject to a change of posture depending on the position adopted by the examined what was determined by the type of chair one was sitting [9]. Weber et al. [10] studied the influence of cervical lordosis position of the head among women (the points A1 and A2). According to the authors of the work, the relationship of measured parameters has been confirmed. This confirms also that the mutual dependence between the positions of the individual characteristic points of the skeleton form a chain with mechanically related items. Pezzan et al. study shows the effect of use of shoes with high heels on the posture of the examined and describes changes being a result of the impact of this factor with the age of the examined people [11]. Other report describes the impact of the frequency of use of shoes with high heels on a change of body posture [12]. In accordance with the presented results, it can be noted that this type of shoes reduces the lumbar lordosis, while the frequency of use of such footwear does not alter the free body posture. It can be found the dependence of certain factors to change the posture of the examined that occur when working at the computer [13]. The incidence of deviations attitude of internal TMJ disorders within the jaw region was not confirmed [14]. Raine and Twomey described dependence of thoracic kyphosis in relation to the position of the shoulders and head [15]. The study was conducted on a sample of 39 examined. In consisted measuring of upper and lower curvature (C7Th6 and Th6-Th12) using the profile shape of the back determined with the help of two-dimensional images taken in sagittal and frontal planes. The images were taken into the computer-assisted process for determination of the position of tags applied to in the important anatomical points of the body of the examined people. The relationship between the position of the head in the sagittal plane and the curvature of the upper thoracic kyphosis was found in the study. According to the conclusions presented, forward head posture (FHP) has influence

${ }^{*}$ Corresponding author: Marek Sutkowski, Institute of Microelectronics and Optoelectronics, Warsaw University of Technology, Poland, Tel: +48 (22) 234-3652; E-mail: sut@imio.pw.edu.pl

Received June 12, 2017; Accepted July 28, 2017; Published July 31, 2017

Citation: Pasko S, Sutkowski M, Zuk B (2017) Evaluation of the Selected Parameters of the Body onto the Location of the Gravity Center Projected on Foot J Spine 6: 380. doi: 10.4172/2165-7939.1000380

Copyright: ( 2017 Pasko S, et al. This is an open-access article distributed under the terms of the Creative Commons Attribution License, which permits unrestricted use, distribution, and reproduction in any medium, provided the original author and source are credited. 
onto the curvature of the upper thoracic kyphosis (C7-Th6), which confirmed the clinical observations reported by the Darnell, Kendall, Mannheimer et al [16-18]. It also asserted the dependence of the position of the head in the Frankfurt plane and the sagittal position of the arms. The examined ones with aspirations to keep the head high in straighten position and stretched upper part of the thoracic part of the spine, have also shown a tendency to offset their arm position to the front. However, the paper did not link presented results with clinical significance. Such a dependence has also not been confirmed in later studies on a larger group of examined (160 people) [19]. These studies were carried out using only the power analysis of selected anatomical points on the basis of a two-dimensional image registration. The fact that no spatial position of points was determined in the process, could have an impact on the discussed result. It is reasonable to use the method for the analysis of three-dimensional data $(\mathrm{x}, \mathrm{y}, \mathrm{z})$ in such studies. Using markers placed on the characteristic points of the human body, e.g., corresponding to the positions of outermost points of the nuchal lower limits, the spinous processes of the vertebrae $\mathrm{C} 7, \mathrm{Th} 1, \mathrm{Th} 6, \mathrm{Th} 7$ and the position of the upper blade angles, allow to quantitative determination of the relative positions of the test points in three-dimensional space. These data, in turn, will be used to determine the geometrical quantities characterizing the attitude of the subject.

\section{The Goal}

The goal of the presented work is to evaluate body posture in coronal plane observed from the back. In the research, a series of selected anatomical points were complying. The nature of human movement apparatus can be understood as a chain connected mechanical components. During the studies on the position of the upper part of the human body it was decided to complete the analysis of the position with the projection of center of body gravity point on the foot. It was determined with use of the position of the ear canal in the sagittal plane with respect to the length of the foot of the examined. The research carried out here concerned the determination of relationships and variability of parameters for habitual (normal) and straighten posture ("at attention", understand as actively corrected). An adequate method of measurement of spatial position of the body characteristic points with the use of markers were adopted by Sutkowski et al. [20].

\section{Methods}

In clinical terms, for determination of the parameters of the attitude of a human body, scoliometers and plurimeters are commonly used as measuring instruments. However, this method does not allow to determine the real values of the positions of the vertebrae, rotation angles and curves for standing, relaxed positions of the examined ones and it changes over time. These changes can be understood as a result of the natural mechanical activity of the body, such as: a small mechanical movements attitudes resulting from human physiology or a change of attitude depending on the momentary alignment of various parts of the body, and finally adopting different body position than the habitual.

There are known methods of non-invasive, non-contact measurement of parameters of human attitude and one of the most important branches are optical methods of computer-assisted analysis. Among them, the most commonly found in the literature is the method of projection stripes and moire [21-23] and photogrammetric [24-27]. The advantages of non-contact systems that do not require application any tags to the body of an examined person are undeniable, and one of the most important is the ease of use and not dependent on individual factors of the person performing the measurement. However, the biggest drawback is the ambiguity of determining the characteristic points of the skeleton, which are made only based on data on body surface area. These points can be draped over the body of the examined, among others, as contrasting with the balls placed on the pins [24] or tags applied by marker pens directly to the skin of the examined [21]. On the other hand, Iunes et al, showed no relationship between the assessment of attitudes carried out visually and measurements made using photogrammetric methods [26].

Thus, in the presented work, an optical method of measurements of the selected body points was used. Characteristic body points were identified by palpation method and marked with a dedicated marking points. This allow to perform analysis of the posture and its changes in time.

\section{Measurement Set-up}

The premise of the measurement system used in the present study was to perform non-invasive measurement which can allow to determine the 3D coordinates of the measurement points: the position of the spinous processes of the vertebrae C7, Th1, Th6, Th7 and the position of the upper blade angles. In addition, a position of the roll center of gravity of the body on foot was also measured by the non-invasive method. The center of gravity was assumed here as geometrical projection on the foot of the point corresponding to the position of ear canal.

In the system for position measurements, a method based on a widely known technique of photogrammetric registration of stereoscopic images (stereopairs) was adopted. All images were recorded with the use of the photographic cameras with high resolution imagers. In the set-up, two digital cameras equipped with CMOS imaging sensors, APS-C format with a resolution of approx, 16 million of effective pixels, equipped with optics with a focal length of $30 \mathrm{~mm}$ were used. Both cameras are mutually synchronized and located symmetrically with respect to the $\mathrm{z}$ axis, with the base of the stereoscopic set-up of 350 $\mathrm{mm}$. Images were recorded in RAW format, and then on the basis of such stored image data a conversion to grayscale-type was made using specialized software Adobe Lightroom. Prepared images were subjected to analyze specifically for the purposes of this study created application (in $\mathrm{C}++$ that uses OpenCV libraries).

Markers in the form of a circle with a diameter of $\phi 10 \mathrm{~mm}$ black-white edges and the center (approx. $\phi 3 \mathrm{~mm}$ ) were glued to the examined skin. As a result, it was possible to quick and unambiguous determination of the center of the marker. The measurement system was prepared for the object distance approx. $1500 \mathrm{~mm}$, the area of measurement space was approx. $600 \mathrm{~mm} \times 1200 \mathrm{~mm} \times 500 \mathrm{~mm}(\mathrm{x}, \mathrm{y}, \mathrm{z})$ and was calibrated by the use of a standard test chart (checkered black and white squares with dimensions of $50 \mathrm{~mm} \times 50 \mathrm{~mm}$ ). With a choice of two images of a stereo pair, the position of the measuring points in the form of coordinates $\left(\mathrm{p}_{\mathrm{x}}, \mathrm{p}_{\mathrm{y}}, \mathrm{p}_{\mathrm{z}}\right)$ were determined.

Each examined person with set of the markers applied on one's body was placed within the designated, calibrated measurement space, with the back directed to the lenses of the measuring system. Examined people/patients were dressed in underwear that does not interfere with the location of the applied markers. The hair was tied at the top of the head and secured with a cap. Tags corresponding to the position of the ear canal were applied directly on the surface of the cap (Figure 1).

Designation of the center of gravity line was made on the basis of geometric analysis of the 2D grayscale image taken in the sagittal plane of the examined one. This method also uses the above described markers, in order to determine the location of desired data points. For this purpose, a digital camera with CMOS type sensor, full frame format (standard 135), with a resolution of approx. 24 million of effective pixels, equipped with a lens with a focal length of approx. 35 
Citation: Pasko S, Sutkowski M, Zuk B (2017) Evaluation of the Selected Parameters of the Body onto the Location of the Gravity Center Projected on Foot. J Spine 6: 380. doi: 10.4172/2165-7939.1000380

Page 3 of 5

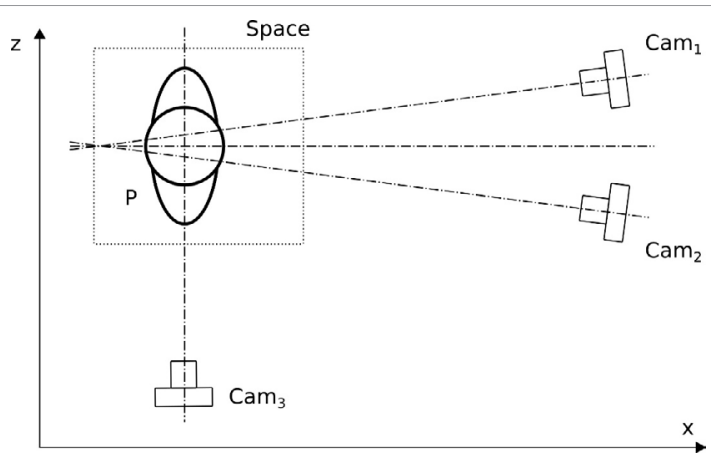

Figure 1: Measurement set-up. Coordinates $x, z$ as on the axes, axis $y$ is perpendicular to the drawing plane. Space-calibrated measuring space (as described in text), P-examined under measurement, Cam1, Cam2photogrammetric streopair of digital imagers, Cam3-coronal plane view camera used for measuring of the examined' body gravity point
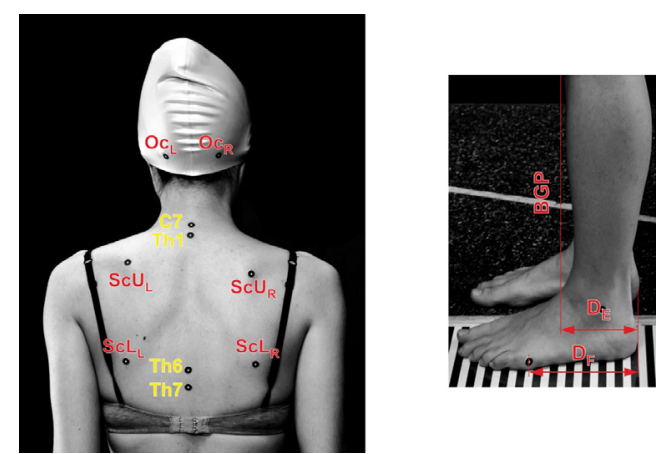

Figure 2: Measured points marked on the examined' body: processus mastoideus-OcL and OcR, angulus superior scapulae-ScUL and ScUR, L and $\mathrm{R}$ indices used as left and right respectively. BGP-projection of the body gravity point.

$\mathrm{mm}$ (locked position of the zoom) was used. Images were recorded in RAW format and the rest of the processing data were the same as described above (Figure 2).

\section{Results}

On the basis of the collected photogrammetric data a determination of $3 \mathrm{D}$ coordinates [26] of the selected characteristic points which were determined as follows: outermost points of the nuchal lower limits-Oc (left), $\mathrm{Oc}_{\mathrm{R}}$ (right); the spinous processes of the vertebrae: $\mathrm{C} 7$, Th1, Th6, $\mathrm{Th} 7$; the position of the upper blade angles-ScU $\mathrm{L}_{\mathrm{L}}$ (left), $\mathrm{Sc}_{\mathrm{R}}$ (right). Based on these points, a set of vectors were calculated:

$$
\begin{aligned}
& \overline{O c}=O c_{L}-O c_{R} \\
& \overline{S c U}=S c U_{L}-S c U_{R} \\
& \overline{C T}=C 7-T h 1 \\
& \overline{T T}=T h 6-T h 7
\end{aligned}
$$

Using the additional data collected during the measurement (plumb line), specified vector indicating vertical in the system was defined. For the study, it was defined five parameters, of which the first four was calculated on the basis of the above-mentioned vectors.

$$
\begin{aligned}
& P_{1}=180-\Varangle(\overline{C T}, \overline{T T}) \\
& P_{2}=\Varangle_{x z}(\overline{O C}, \overline{S c U})
\end{aligned}
$$

$$
\begin{aligned}
& P_{3}=\Varangle\left(\overline{C T}, \bar{V}_{t}\right) \\
& P_{:}=\Varangle\left(\overline{T T}, \bar{V}_{t}\right)
\end{aligned}
$$

And, at least, the fifth parameter was calculated with use of images showing coronal plane, as the ratio of the vertical projection of the center of the ear canal from the heel to the length of the foot:

$$
P_{5}=100 \cdot\left(\frac{D_{E}}{D_{F}}\right)
$$

where $\mathrm{D}_{\mathrm{E}}$-distance to the finger bones, $\mathrm{D}_{\mathrm{F}}$-distance of the projection of the body gravity point in relation to the heel.

The parameters $\mathrm{P}_{1}$ to $\mathrm{P}_{4}$ are given in degrees and $\mathrm{P}_{5}$ as a percentage. The values obtained for the parameters are shown in Figure 3.

The data of all the distributions was tested with Shapiro-Wilk test [28], confirming that they have an approximately normal distribution. Then $\mathrm{P}_{5}$ parameter was correlated with other parameters using the r-Pearson correlation yielding the results presented in Table 1 .
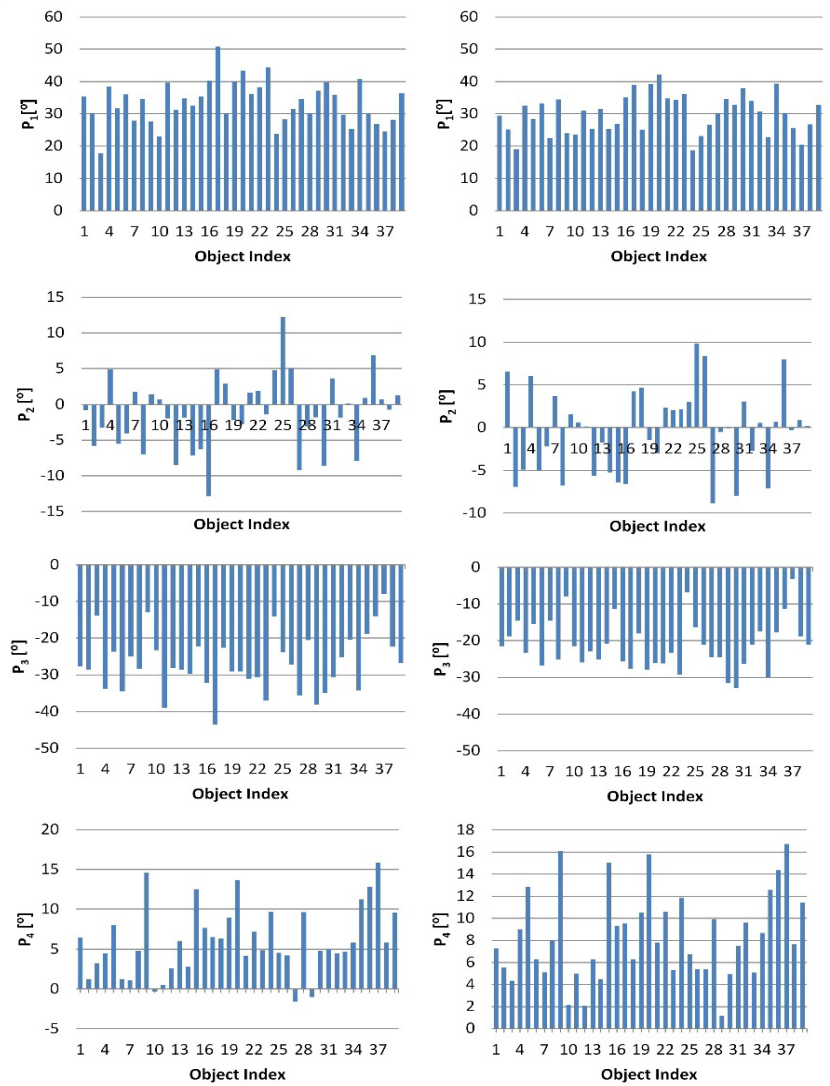
Object Index

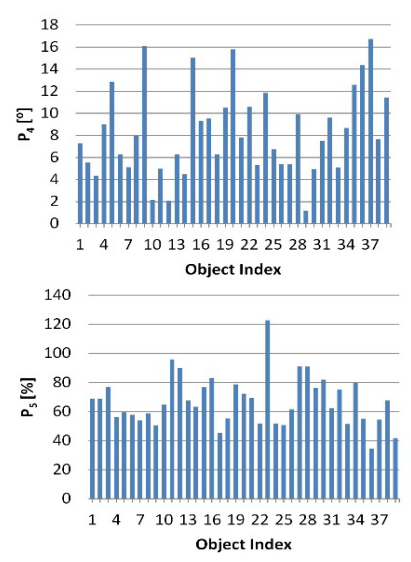

Figure 3: The values of the parameters $\mathrm{P} 1$ to $\mathrm{P} 5$ assigned to each examined of research for a habitual posture (a, c, e, g, i respectively) and the straighten one (b, $d, f, h$, j respectively). 


\begin{tabular}{|c|c|c|c|c|}
\hline \multirow{2}{*}{} & \multicolumn{2}{|c|}{ Habitual posture } & \multicolumn{2}{c|}{ Straighten posture } \\
\cline { 2 - 5 } & $\boldsymbol{r}$ & $\boldsymbol{p}$-value & $\boldsymbol{r}$ & $\boldsymbol{p}$-value \\
\hline$P 1$ & 0.43 & 0.006 & 0.33 & 0.042 \\
\hline$P 2$ & -0.51 & 0.001 & -0.45 & 0.004 \\
\hline$P 3$ & -0.62 & 0.001 & -0.50 & 0.001 \\
\hline$P 4$ & -0.50 & 0.001 & -0.37 & 0.022 \\
\hline
\end{tabular}

Table 1: Correlation of the P1-P4 parameters in relation to P5. $r$-the Pearson correlation coefficient, $p$-value-the statistical significance level.

It was adopted by Cohen [29] the following assessment of the strength of the correlation:

$0.1<|\mathrm{r}|<0.3$-low correlation,

$0.3<|\mathrm{r}|<0.5$-average/moderate.

$|\mathrm{r}|>0.5$-high/strong correlation.

On the basis of this distinction it can be concluded that the parameters $\mathrm{P}_{1}$ to $\mathrm{P}_{4}$ are correlated with a parameter $\mathrm{P}_{5}$ with the level of at least moderate, wherein the parameter $P_{1}$ has positive relationship with the parameter $\mathrm{P}_{5}$ and the rest are in relation to it in negative relationship. It can be seen that parameter $\mathrm{P}_{2}$ (showing mutual orientation of the lines designated by two outermost points of the nuchal lower limits $\left(\mathrm{Oc}_{\mathrm{L}}, \mathrm{Oc}_{\mathrm{R}}\right)$ and the line designated by two points of upper blade angles $\left.\left(\mathrm{Sc}_{\mathrm{I}}, \mathrm{Sc}_{\mathrm{R}}\right)-(1.1),(1.2),(2.2)\right)$ and $\mathrm{P}_{5}$ (showing relative position of the body gravity point projected onto foot-(2.5)) have stronger mutual relationship than relationship of parameters $\mathrm{P}_{1}$ (2.1) and $\mathrm{P}_{4}(2.4)$ to parameter $\mathrm{P}_{5}$. The strongest relationship is observed for parameter $\mathrm{P}_{3}(2.3)$.

\section{Discussion}

The analysis of body posture or an individual's way of "keeping up" is the first step in a clinical study of people with various ailments of the musculoskeletal system. Nowadays, according to the principles of Evidence Basic Medicine (EBM), more and more attention is paid to the imaging disorders and/or asymmetry of the body with the use of diverse diagnostic equipment. The paper presents a pilot study using the photogrammetric system to assess the asymmetry of the cervical triangle of setting the upper blade angles, and the impact of these changes on a vertical lateral derived from the opening of the external ear canal. It was measured in two positions: habitual (normal) and the straighten one (so-called "at attention") in both cases with one's eyes directed at one place. The subjects were 39 young healthy people, with no neurological disorders and somatic diseases, but with different types of feet, occasionally pre-existing injuries of the lower limbs. Subsequently, it showed the extreme points of asymmetry nuchal lower limits in relation to the line formed at the height of the upper blade angles, and the vertical projection parameter foot (parameter $\mathrm{P}_{2}$ ). This interesting observation highlights the essence of the asymmetry, because the same absolute angle position of the points of a triangle neck with the location of the vertical line at the rate of no correlation (parameter $\mathrm{P}_{3}$ ). This type of relationship seems to be measurable confirmation of myofascial meridians presented by Myers [30]. The spiral line, because it is primarily concerned with this type of asymmetry of the body, entwines body-on both sides, in the shape of a double helix. It starts at or above the limits nuchal successively goes down to the muscle layered head, layered neck and connects to the spinous processes of the vertebrae C6 to Th7. Then it combines with a parallelogram muscle larger and smaller on the opposite side of the body (and the deeply located muscle front gear) from attaching to the medial edge of the blade. The further course of the tape refers to the oblique abdominal muscles internal and external, whose trailer bone are in the region of the iliac crest and the anterior superior iliac spine. From this point, the spiral line returns to the starting side of the body, and through the muscle tensor fascia lata and the band ilio-tibial guided by the so-called stirrup created by fascial and mechanical connection between the tibialis anterior muscle and tibial long, affecting setting foot on the changes in the cervical triangle. Then, through the biceps femoris, sacrotuberous ligament, fascia crosslumbar erector spinae muscles return to the occipital protuberance. This combination of a number of structures of myofascial structures draws attention to the multiplicity of mechanisms contributing to the cause of asymmetry in body posture and the concomitant diagnostic difficulties static posture [31]. In the physiotherapeutical literature, a method of evaluation of the body posture similar to presented in this paper was never mentioned. Characteristic anatomical points marked in this research placed on the skull (the outermost points of the superior nuchal line of the skull) were never analyzed in reference to determination of the spatial position of the human head.

The obtained data clearly show a stronger correlation in both the attitudes of the body between the angles set of vectors C7/Th1 and Th6/ Th7 onto the foot than asymmetric triangle neck with the parameters of the angle of the head in space (parameters $\mathrm{P}_{3}, \mathrm{P}_{4}$ ).

Regarding the effect of the study-the method used to describe the portion of the figure, the mechanics of posture, pay attention to the importance of compensation patterns in the body. As it is known, the core of the body posture, organ axial segmental construction is the backbone of the flexible intervertebral discs, compared to two inverted pendulums. Upper pendulum head is mounted on a short, but very movable cervical and bottom-a little movable thoracic (with chest) adjacent to a moving segment of the lumbar spine based on the pelvis. By the chest and shoulder girdle, as well as through the pelvis and lower extremities, spine directly or indirectly involved in almost every activity of man. "Rigging" skeletal muscle structures skeletal system by myofascial structures affect the protective function of the spine, namely the physiological curvature. Although these pilot studies illustrate only the upper part of the body posture changes and coexistence with the distribution line vertical lateral foot and ignore the relationship settings pelvis in space, they demonstrate the ability to assess the dislocation of selected segments of the body using photogrammetric techniques.

The advantage of this type of tool is also an opportunity to present the changes in body shape due to targeted health training or the therapy, as a motivating factor to change the incorrect habits. Analyzing the given results can be seen that it is impossible to determine the correlation of the absolute values of the angles of rotation of simple subject to analysis. To demonstrate the interdependence, it is necessary to take into account the sign of the angle of rotation simple. This indicates a significant impact on the attitude of asymmetry obtained as the results of this study. The causes for the existing asymmetry indicates the most common disorders of the organs of hearing, sight, right- or left-handedness, or, finally, occupational factors. The direction of the asymmetry has an important diagnostic and therapeutic. The use of measurement methods which give results including directional asymmetry in an easy and visual way to prove examinee progress of therapy and will motivate for further action.

The P5 factor denotes a projection of the body gravity center projected on foot (taken as the position of the ear canal) [1,3]. It was used as a base for statistical analysis of all other parameters calculated in the experiment.

\section{Limitations}

Presented study has some limitations which should be considered in further research. Results and analysis shown in the paper have pilot 
character and there are many factors which corresponds between body posture and spatial position of the head which was omitted and should be analyzed in further work. The possible reason of the asymmetry of the upper body part can be dysfunctions or disorders of hearing or sight, occlusion defects or "left-/right-sided" body. None of the examined volunteers has noticed such features. Moreover, a character of the professional activity can play a role in this question. Thus, continue of this topic with use of the applied measurement system is strongly desirable and will be performed in near future.

\section{Conclusion}

Results presented in the paper can be concluded as follows:

1. The angle of upper vertebral kyphosis in the sagittal plane (parameter $\mathrm{P}_{1}$ ) is proportionally correlated to the position of the body gravity point projected on foot (parameter $\mathrm{P}_{5}$ ). It can be explained by corresponding directions of the lean of the body and relative position of the body gravity point projected on foot. The change of the correlation value of these two factors $\left(\mathrm{P}_{1}\right.$ and $\left.\mathrm{P}_{5}\right)$ for the undefined active position (straighten, actively corrected) proves that examined people has activated intentionally his/her postural muscles. In effect, the scapulae were pulledoff, the kyphosis was decreased and in consequence the position of the body gravity point projected on foot was moved to the heels.

2. Parameter $\mathrm{P}_{2}$ (the angle between the lines defined by $\mathrm{Oc}_{\mathrm{L}}, \mathrm{Oc}_{\mathrm{R}}$ and $\mathrm{ScU}_{\mathrm{L}}, \mathrm{ScU}_{\mathrm{R}}$ points) has negative correlation with $\mathrm{P}_{5}$. It can be explained by possible asymmetries of the tensions in the upper part of the body and the position of the body gravity point projected on foot. Small differences between two postures adopted in the studies proves that intuitive activation of the postural muscles has no (or small) influence for this asymmetry.

3. Correlation of parameters $\mathrm{P}_{3}$ and $\mathrm{P}_{4}$ (describing respectively: angle between $\overline{T T}-\bar{V}_{t}$ vectors and $\frac{3}{T T}-\bar{V}_{t}$ vectors in the coronal plane looking from the back) with parameter $\mathrm{P}_{5}$ are negative. The values of these correlations $\left(\mathrm{P}_{3}-\mathrm{P}_{5}\right.$ and $\left.\mathrm{P}_{4}-\mathrm{P}_{5}\right)$ for both adopted positions (habitual and straighten one) differ very strongly. It can be explained as a cumulative effect of moving the head to the front, decreasing of the vertebral kyphosis and moving of the projection of the body gravity point back, to the heels.

4. The habitual posture is natural, free and thus is very often loaded with errors, deviations from the values recognized as a standard. Moreover, it can be explanation for higher values of all correlations for habitual posture than for the straighten one.

5. In general, higher values of the p-values obtained during analysis for the straighten position than for the habitual one denotes higher statistical significance of the results measured for straighten posture (actively corrected).

\section{References}

1. Kapandji Al (2008) The physiology of the joints, volume 3: The spinal column, pelvic girdle and head. (6th edn) Elsevier Health Sciences, Netherlands.

2. Neumann DA (2010) Kinesiology of the musculoskeletal system: Foundations for rehabilitation (2nd edn) Mosby Elsevier, USA.

3. Chaitov L (2015) Positional release techniques (4th edn) Elsevier, Netherlands.

4. Błaszczyk J (2011) Biomechanika kliniczna. Wydawnictwo Lekarskie PZWL.

5. Neumann DA (2010) Kinesiology of the musculoskeletal system: Foundations for rehabilitation. (2nd edn). Mosby Elsevier, USA.

6. Nordin M, Frankel VH (2012) Basic biomechanics of the musculoskeletal system, (4th edn). Lippincott Williams \& Wilkins, USA.

7. Cheng HY, Lien YJ, Yu YC, Ju YY, Pei YC, et al. (2013) The effect of lower body stabilization and different writing tools on writing biomechanics in children with cerebral palsy. Res Dev Disabil 34: 1152-1159.
8. Fortin C, Ehrmann Feldman D, Cheriet F, Labelle H (2013) Differences in standing and sitting postures of youth with idiopathic scoliosis from quantitative analysis of digital photographs. Phys Occup Ther Pediatr 33: 313-326

9. Annetts S, Coales P, Colville R, Mistry D, Moles K, et al. (2012) A pilot investigation into the effects of different office chairs on spinal angles. Eur Spine J 21 Suppl 2: 165-170.

10. Correa ECR, Milanesi JM, Soares JC, Trevisa ME (2012) Craniocervical posture: Cephalometric and biophotogrammetric analysis. Braz J Oral Sci 11: 416-421.

11. Pezzan PA, Joao SM, Ribeiro AP, Manfio EF (2011) Postural assessment of lumbar lordosis and pelvic alignment angles in adolescent users and nonusers of high-heeled shoes. J Manipulative Physiol Ther 34: 614-621.

12. lunes DH, Monte-Raso VV, Santos CBA, Castro FA, Salgado HS (2008) Postural influence of high heels among adult women: Analysis by computerized photogrammetry. Braz J Phys Ther 12: 454-459.

13. Straker LM, O'Sullivan PB, Smith A, Perry M (2007) Computer use and habitual spinal posture in Australian adolescents. Public Health Rep 122: 634-643.

14. Munhoz WC, Marques AP, De Siqueira JT (2005) Evaluation of body posture in individuals with internal temporomandibular joint derangement. Cranio 23 : 269-277.

15. Raine S, Twomey L (1994) Posture of the head, shoulders and thoracic spine in comfortable erect standing. Aust J Physiother 40: 25-32.

16. Darnell MW (1983) A proposed chronology of events for forward head posture. J Craniomandibular Pract 1: 49-54.

17. Kendall FP, McCreary EK (1984) Muscles, testing and function. (3rd edn) Brit J Sport Med 18:25.

18. Mannheimer JS, Rosenthal RM (1991) Acute and chronic postural abnormalities as related to craniofacial pain and temporomandibular disorders. Dent Clin N Am 35: 185-208.

19. Raine S, Twomey L (1997) Head and shoulder posture variations in 160 asymptomatic women and men. Arch Phys Med Rehabil 78: 1215-1223.

20. Sutkowski M, Paśko S, Żuk B (2017) A study of interdependence of geometry of the nuchal neck triangle and cervical spine line in the habitual and straightened postures. J Anat Soc India 66: 31-36.

21. Drzał-Grabiec J, Rachwał M, Podgorska-Bednarz J, Rykała J, Snela S, et al (2014) The effect of spinal curvature on the photogrammetric assessment on static balance in elderly women. BMC Musculoskelet Disor 15:186.

22. Drerup B, Hierholzer E (1987) Shape analysis of the back surface: measurement of the lateral tilting of the pelvis from posterior iliac spines. In: Stokes, IAF (Hrsg.). Surface topography and spinal deformity IV: 275-282. Gustav FischerVerlag, Stuttgart, New York, USA

23. Glinkowski W, Michoński J, Glinkowska B, Sitnik R (2013) Manual versus automatic indices assessment based on a marker-less, three dimensional, structural light surface topography postural evaluation. Preliminary study, Scoliosis 8 (Suppl 1): O24.

24. Furlanetto TS, Sedrez JA, Candotti CT, Loss JF (2016) Photogrammetry as a tool for the postural evaluation of the spine: A systematic review. World J Orthop 7: $136-148$.

25. Patias $P$, Stylianidis E, Pateraki M, Chrysanthou G, Contozis C, et al. (2006) 3D digital photogrammetric reconstructions for scoliosis screening. Proceedings of the ISPRS Com. V Symposium. The International archives of the photogrammetry, remote sensing and spatial information sciences XXXV (Part 5), Dresden, Germany.

26. Iunes DH, Bevilaqua-Grossi D, Oliveira AS, Castro FA, Salgado HS (2009) Comparative analysis between visual and computerized photogrammetry postural assessment. Brazilian Journal of Physical Therapy 13.

27. Berryman F, Pynsent P, Fairbank J, Disney Sm (2008) A new system for measuring 3D back shape in scoliosis. Eur Spine J 17: 663-672.

28. Shapiro SS, Wilk MB (1965) An analysis of variance test for normality (complete samples). Biometrika 52: 591-611.

29. Cohen J (1988) Statistical power analysis for the behavioral sciences. (2nd edn), Lawrence Erlbaum publishing, New Jersey, USA.

30. Myers TW (2010) Taśmy anatomiczne. Meridiany Mięśniowo-Powięziowe dla Terapeutow Manualnych i Specjalistow Leczenia Ruchem. DB Publishing Warszawa, Poland.

31. Chaitow L (2014) Fascial dysfunction: Manual therapy approaches. Handspring Publishing, Scotland, UK. 